\title{
El enfoque por tareas en la enseñanza/aprendizaje del francés lengua extranjera: una experiencia para la reflexión
}

\author{
Marienma García, Concha Prieto y M. J Jesús Santos
}

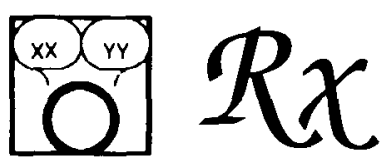

El enfoque por tareas en la enseñanza de la lengua extranjera ba recibido una gran atención en los últimos años. Sin embargo, existen pocos trabajos que, desde la propia práctica educativa, reflexionen sobre sus ventajas e inconvenientes. La experiencia que presentan las autoras en este artículo llena esta laguna.

\section{INTRODUCCION}

El enfoque por tareas como opción metodológica se perfila a lo largo de los años 80 dentro del enfoque comunicativo del que recoge principalmente la centración en el alumno y el concepto de competencia comunicativa. El alumno, protagonista y responsable de su propio aprendizaje, impone sus estrategias y modos preferidos de trabajo mediante la negociación que estará presente durante todo el proceso de enseñanza/aprendizaje. Habrá que determinar, como dice Breen:

«[...] quién hace qué, con quién, sobre qué, con qué recursos/medios/apoyos, cuándo, por cuánto tiempo, cómo y con qué objetivo pedagógico.» (Breen 1987:25)

\section{COMPONENTES DEL ENFOQUE}

Tal y como su nombre indica, el enfoque por tareas está basado en un conjunto de tareas organizadas en torno a un tema y no en torno a un objetivo lingüístico concreto: importa así más el significado que la forma.

El proceso pedagógico se planifica en unidades temáticas que tienen como objetivo-meta la realización de una tarea final relacionada con el tema en cuestión.

La unidad temática comienza con la elección del tema que es el principio-guía. Debe elegirse partiendo de los intereses de los alumnos y de su nivel de conocimientos. De este modo, se garantiza una mayor motivación e implicación en el proceso. En la negociación con los alumnos, habrá que considerar que dicho tema deberá ir ligado a su experiencia personal y a su conocimiento del mundo, pero además, habrá que tener en cuenta las circunstancias y condicionantes de la situación de enseñanza/aprendizaje. 
En cuanto a los objetivos, se contempla, por una parte, alcanzar una competencia comunicativa mediante la integración de las cuatro destrezas en la realización de la tarea final; $y$, por otra, se persigue un objetivo de autonomía del aprendizaje que desarrolle la capacidad de «aprender a aprender» la nueva lengua y a aplicar dentro y fuera del contexto educativo lo aprendido en el aula (Holec 1983:88). De este modo, el alumno reflexiona sobre su propia comunicación y sobre las capacidades y estrategias que pone en juego. El hecho de que los alumnos conozcan los objetivos a conseguir afianza su responsabilidad en el propio aprendizaje y facilita la auto-evaluación.

El contenido de los programas basados en tareas es el propio proceso de aprendizaje. En palabras de Estaire y Zanón (1990), la tarea final constituye el vértice en el que confluyen todos los componentes del enfoque y la manifestación de «mayor potencial comunicativo» ya que representa la competencia comunicativa en su versión multidisciplinar. Para llevar a cabo dicha tarea final serán necesarias unas actividades facilitadoras o subtareas que pueden ser tanto comunicativas como de aprendizaje. Los componentes temáticos y lingüísticos que intervienen son los resultantes de dichas tareas.

Continuando con los conceptos desarrollados por Estaire y Zanón (opus cit.), una tarea comunicativa debe representar los procesos de comunicación de la vida real, centrándose en la negociación del significado a través de la comunicación hablada y/o escrita de la lengua que se aprende.

Las tareas de aprendizaje se seleccionan según criterios de metacomunicación ya que servirán para proporcionar a los alumnos los conocimientos necesarios para la realización de las tareas comunicativas.

De las tareas comunicativas y de aprendizaje se deriva el desarrollo en el alumno de una serie de capacidades por la aplicación de las cuatro destrezas. Estas capacidades se traducen en interpretar, expresar y negociar el significado.

En el enfoque por tareas, a diferencia de otro tipo de metodología, los contenidos a trabajar no se determinan previamente sino tras el análisis de la tarea final de la unidad. Los componentes serán de dos tipos: temáticos (aspectos que afectan al tema elegido) y lingüísticos (nociones, funciones, estructuras, léxico, fonética, etc.) necesarios para realizar las tareas finales.

Una de las características de los programas basados en tareas es que tanto los componentes temáticos como lingüísticos se abordan de forma global y cíclica.

La secuenciación de los contenidos se hace atendiendo a la dificultad de las tareas, según el grado de competencia comunicativa que poseen los alumnos y según los condicionantes y problemas que se preven en la puesta en práctica del programa.

En cuanto a los materiales, entendemos como tales todos aquellos documentos y soportes que presentan la lengua como instrumento de comunicación y favorecen la implicación del alumno en su propio aprendizaje.

Dada la gran diversidad de medios para transmitir y acceder a la información, será necesario presentar los materiales de la forma lo más auténtica posible, utilizando el medio para el que naturalmente fueron creados (radio, TV, periódico, etc.), precisamente porque los diferentes canales de información influyen de manera determinante en la lengua que los pone de manifiesto. Por otra parte, esa riqueza y diversidad multimedia favorece el descubrimiento de nuevas estrategias de aprendizaje. Al variar las fuentes de información (visual, auditiva, kinésica), se atiende a los diferentes estilos cognitivos de cada alumno.

Precisamente el enfoque por tareas, dado su carácter flexible y abierto, permite además la diferenciación de niveles en la consecución de objetivos porque ofrece cantidad y variedad de actividades y de materiales.

Metodológicamente, esta variedad conlleva la necesidad de trabajar en grupo para cooperar en la realización de la tarea. Dentro del grupo, cada alumno aporta sus 
propias destrezas y conocimientos al resultado final, a la vez que contribuye a su autonomía al trabajar con sus propias técnicas y estrategias de aprendizaje.

La evaluación en el enfoque por tareas se contempla no como algo externo al proceso de aprendizaje sino como parte integrante del mismo. El funcionamiento y los resultados de cada tarea se incorporan a dicho proceso y es posible, de este modo, realizar una evaluación contínua de cada tarea/subtarea/etapa. Nos encontramos, pues, con una evaluación contínua, procesual, formativa y sumativa que informa tanto al profesor como al alumno de todos y cada uno de los componentes del proceso.

El alumno podrá evaluar aspectos como:

-su trabajo individual;

—u trabajo como miembro del grupo;

—el trabajo de sus compañeros y del profesor;

-los materiales utilizados y los procedimientos seguidos;

-los resultados obtenidos.

Al ser el alumno centro del proceso de aprendizaje participa en la toma de decisiones relativas a dicho proceso y en la evaluación del mismo. Será además el corresponsable de todas las decisiones negociadas, aportando igualmente materiales de trabajo. Esta toma de decisiones y evaluaciones obliga al alumno a reflexionar sobre su propio aprendizaje, sus problemas en el mismo y sus gustos y necesidades.

Por su parte, el profesor abandona su papel central y se convierte en corresponsable con el alumno, en facilitador y organizador de los recursos, en animador y estimulador del trabajo en los grupos, monitorizando y reconduciendo las actividades hacia los objetivos propuestos. Sin embargo, sigue siendo responsable absoluto en la elección de los componentes lingüísticos.

\section{NUESTRA EXPERIENCIA}

El enfoque por tareas ha sido aplicado en la E.U. de Educación de Palencia, en la materia de «Francés», optativa que se imparte en $2^{\circ}$ curso de la especialidad de Educación Física.

Consideramos que la metodología propuesta por el enfoque por tareas podía ajustarse al público con el que nos encontramos en esta asignatura, ya que se trataba de un grupo muy heterogéneo, con distintos niveles de lengua, con una personalidad atípica, por estar acostumbrados a una constante actividad y tener necesidad de acción.

En cuanto a sus necesidades de lengua francesa en el desarrollo de su profesión contemplamos tanto las que surgirían de una posible consulta bibliográfica, como aquellas en que la lengua actuaría de medio de comunicación en posibles intercambios con centros escolares franceses.

La metodología por la que tendríamos que optar debería presentar unas características que se asimilasen a las del enfoque por tareas ya que necesitábamos:

—que se centrara en los alumnos, partiendo de sus necesidades específicas;

-que no impusiera un ritmo de aprendizaje igual para todos sino que permitiera a cada individuo desarrollar sus propias destrezas, favoreciendo una autonomía en el aprendizaje;

—que les diera responsabilidad, haciéndoles co-gestores del proceso de enseñanza/aprendizaje, por medio de la negociación, lo que propiciaría su motivación;

-que favoreciera el aprendizaje significativo, haciendo resaltar la funcionalidad de lo que aprendían;

—que desarrollara las destrezas tanto receptivas como productivas en actividades que las integraran, a un mismo nivel de importancia; 
—que utilizara temas de la vida real y favoreciera la comunicación auténtica y la interacción en el aula;

—que permitiera una cooperación entre los alumnos para que los más avanzados ayudaran a los más atrasados y todos intervinieran según sus capacidades y su conocimiento del mundo;

- que canalizara toda su energía hacia una constante participación y acción.

Como muestra de nuestra experiencia, desarrollamos la unidad temática que comenzamos después de las Navidades y en la que hemos trabajado durante algo más de un mes, a razón de tres horas semanales.

Esta unidad se propuso con el título «Viajar». Nuestros alumnos simulaban ser ya profesores de Educación Física en un centro escolar y se proponían organizar un viaje a una estación de esquí de los Alpes, con su hipotética clase.

Para el diseño de la unidad temática, creamos un modelo basándonos en el propuesto por Estaire y Zanón (opus cit.: 76) que explica claramente la interrelación que existe entre los distintos elementos del proceso, cuyo eje central lo constituyen las tareas finales (ver figura 1 ).

\section{FIGURA 1}

Organización de la unidad temática «Viajar».

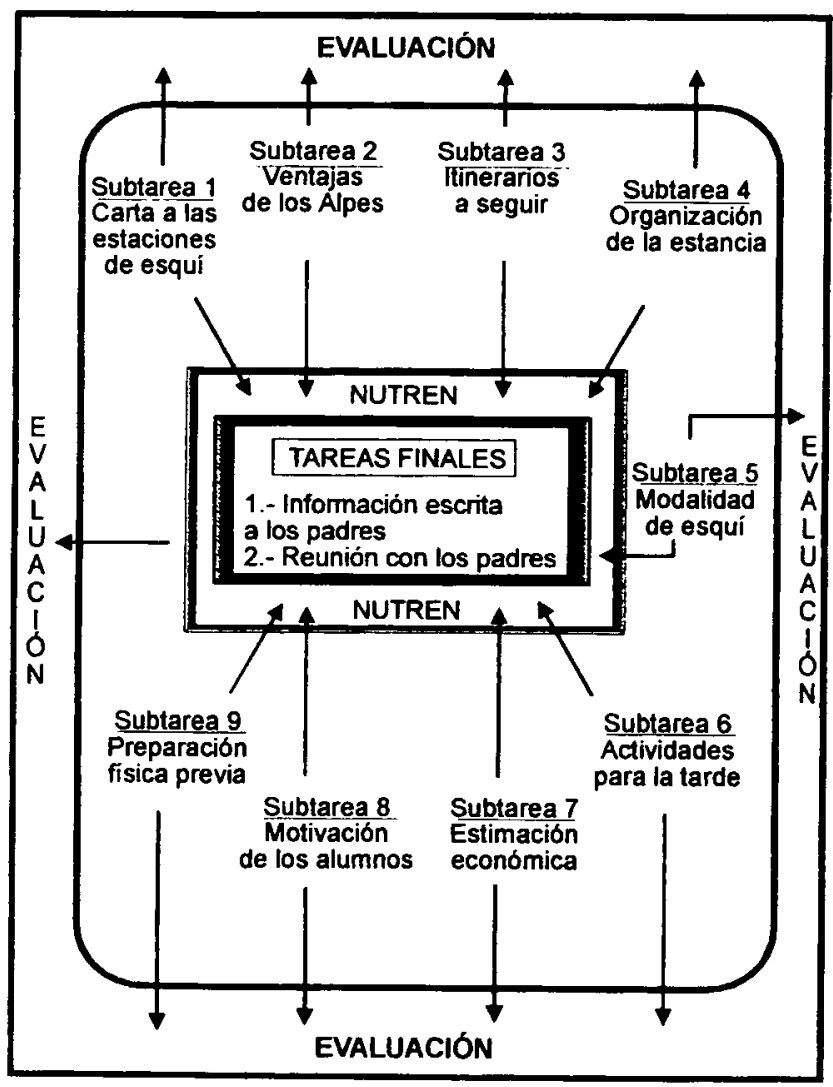

La elección de las tareas finales, primer paso a negociar en el aula, nos permitió determinar los componentes temáticos necesarios para su realización a través de las subtareas. Estos componentes temáticos determinaron a su vez los componentes linguísticos. Tanto unos como otros incidieron en la elección del tipo de subtareas que alimentarían y facilitarían las tareas finales. 
Partiendo, pues, del tema "Viajar», los objetivos que se propusieron se agruparon en comunicativos, culturales y formativos. Como objetivos comunicativos, nos propusimos desarrollar la capacidad de pedir, dar y comprender información, oral y escrita, en relación con la organización de un viaje. Como objetivos culturales: conocer una región francesa y las costumbres en una estación de esquí. Como objetivos formativos: desarrollar estrategias para la organización de viajes al extranjero con un grupo de alumnos, seleccionar ejercicios físicos que fortaleciesen los músculos que intervienen en la práctica de un deporte determinado y conocer técnicas de motivación para la clase.

Estos objetivos debían concretarse en la realización de dos tareas finales: una escrita que consistía en la redacción de una carta o circular dirigida a los padres informando sobre el proyecto de viaje a los Alpes aclarando aspectos organizativos y económicos cuya consigna era: intentar convencer, expresando entusiasmo. Otra oral que consistía en la simulación de una reunión con los padres en la que los profesores deberían contestar a las preguntas que aquellos les formularan sobre el viaje, antes de dar su consentimiento. La consigna era: intentar dar garantías de seguridad y tranquilizar a los padres.

El tema, los objetivos y las tareas finales fueron los únicos puntos que se especificaron desde un principio. Las demás decisiones tomadas durante la Unidad temática fueron negociadas con los alumnos.

El primer punto a negociar fue la determinación de los componentes temáticos necesarios para la realización de las tareas finales. Esto nos permitió predecir los componentes lingüísticos y organizar las subtareas, que resultaron ser nueve.

La primera tarea consistió en solicitar información de distintas estaciones de esqui de los Alpes para conocer las instalaciones, tipos de alojamiento, precios y servicios que ofrecían. Esto nos permitió abordar las técnicas de comunicación epistolar.

Mientras se recibía la información solicitada, se prosiguió con la unidad temática, tratando de descubrir las ventajas que podría ofrecer la práctica de esquí en los Alpes para justificar la excursión ante los padres. Para facilitar esta segunda tarea, se analizaron unos folletos de cursillos de esquí en los Alpes que nos permitieron trabajar la lengua específica y extraer una serie de razones a esgrimir en la reunión final.

La tercera tarea, organización del viaje de ida y vuelta, empezó con la toma de decisión a nivel de pequeño grupo del medio de transporte a utilizar y del itinerario a seguir. Como todos los grupos eligieron el autocar, se distribuyeron mapas de carretera, se calcularon los kilómetros, las paradas, se analizaron folletos de hoteles de carretera, por si se decidía dormir en el camino. El método de trabajo consistió en tomar decisiones a nivel de pequeño grupo y discutirlas posteriormente con el grupo-clase, volviendo al pequeño grupo para negociar las decisiones definitivas.

La cuarta tarea consistió en organizar la estancia. Para ello, se trabajó sobre los folletos que se habían ido recibiendo de las distintas estaciones de esquí. Se establecieron comparaciones en cuanto a prestaciones y a precios y se tomaron decisiones.

La quinta tarea tenía como objetivo decidir la modalidad de esquí que se aconsejaría a los niños. Para ello se trabajó con unas fotocopias sacadas de la revista $L e$ Français dans le Monde (Janvier 1992) donde se describían las diferentes modalidades que intervinieron en las Olimpiadas de Albertville. Se decidió en pequeño grupo la modalidad apropiada para adolescentes debutantes y se discutió posteriormente a nivel de grupo-clase.

La sexta tarea, organización de actividades para la tarde/noche, se hizo en base al input recibido de la ayudante de conversación quien dió una charla sobre las actividades usuales en una estación de esquí francesa, deteniéndose principalmente en la descripción de las «soirées de fondue» $\mathrm{y}$ «de raclette».

La séptima tarea consistió en hacer una estimación económica, con los datos que se tenían y con otros que fue preciso obtener fuera del aula como el precio del autocar por $\mathrm{km}$., el precio de la póliza de seguros, etc. 
La octava tarea tuvo como objetivo la motivación de los alumnos, que se centró básicamente en la decoración de la clase con carteles alusivos al esquí y la montaña. Pretendíamos que nuestros alumnos desarrollaran su imaginación y su creatividad. Como documentos de apoyo, presentamos tarjetas postales humorísticas y un cartel sobre los diez mandamientos del esquiador, en el que se explotaron primero los elementos icónicos y después los lingüísticos.

Para terminar, la novena tarea consistió en seleccionar ejercicios de gimnasia que prepararan a los alumnos para la práctica del esquí, partiendo de un estudio de los músculos que intervienen en este deporte.

Con toda la información extraída de las nueve tareas descritas, nuestros alumnos trabajaron individualmente en la confección de una carta a los padres, donde se informaba del proyecto de viaje de forma detallada. Los criterios de valoración para la evaluación de esta tarea final se negociaron, priorizando la adecuación y riqueza de la lengua utilizada y la capacidad para convencer a los padres, utilizando todo tipo de estrategias. La producción oral de nuestros alumnos durante la reunión con los padres, que fue grabada para que pudieran oirse y auto-corregirse, se evaluó en base a criterios de capacidad de comunicación, riqueza lingüística y adecuación al contexto. Se valoró igualmente el poder de convicción y la capacidad para desenvolverse en situaciones no previstas.

\section{REFLEXION SOBRE LA PRACTICA}

Dado que uno de los principios del Enfoque por Tareas es la contínua evaluación de todos y cada uno de los elementos del programa, este trabajo carecería de coherencia y rigor si no hiciésemos un análisis de la realidad de nuestra experiencia.

En este análisis pudimos observar que la motivación se mantuvo a lo largo de toda la unidad debido, por una parte, a la utilización de la lengua en siruaciones auténticas: escribir cartas a organismos franceses y recibir la información requerida, manejar mapas de carreteras y otros materiales sociales; y por otra parte, al hecho de tener que llevar a cabo un proyecto común a cuya realización estaban encaminados todos los esfuerzos, individuales y del grupo-clase.

El factor de heterogeneidad de conocimientos, que en principio suponía un obstáculo, se pudo superar por la propia metodología de cooperación en la que cada alumno aportó sus capacidades y estrategias, sintiéndose todos útiles, incluso los más desfavorecidos lingüísticamente.

Igualmente se respetó el ritmo de aprendizaje de cada alumno al tener que interpretar los documentos de forma individual. La apropiación de nuevos contenidos se vió favorecida por su contínua reutilización en diferentes contextos y en la realización de la tarea final.

La negociación a la que se sometían todas las decisiones hizo que los alumnos se sintieran responsables de lo que ellos podían aportar al grupo y a la tarea y se implicaron íntegramente.

No obstante, también nos encontramos con una serie de limitaciones y condicionantes a la hora de llevar a cabo nuestro trabajo:

En un principio constatamos una cierta inseguridad ante esta metodología nueva para los alumnos, que les pedía responsabilidad y autonomía en el aprendizaje. Intentamos solventar este problema haciendo reflexiones periódicas sobre lo aprendido e incluso resolviendo las dudas que se presentaban, con actividades de práctica controlada.

Por otra parte, claramente y desde el principio, tuvimos que encauzar los contenidos y las actividades a la realización de las tareas finales, ya que cuando les ofrecíamos más inpur del necesario, la atención y el interés disminuían, al tener dificultades de asimilación y al no ver su utilidad inmediata para la tarea. 
Otra dificultad que surge en la utilización de este enfoque es la limitación de los materiales disponibles. Al no estar en una situación de inmersión en la lengua francesa, la negociación para elegir las tareas casi siempre resulta mediatizada por esta limitación. Así en la elección del «Viaje a los Alpes» influyó considerablemente el hecho de que se dispusiera de bastante información sobre el tema, si bien, otra buena parte de dicha información fué adquirida por los propios alumnos a lo largo de la realización del proyecto.

En cuanto a la utilización de la lengua extranjera (Francés) por nuestros alumnos, resultó bastante difícil durante la fase de negociación y sobre todo cuando se comunicaban entre ellos. La falta de nivel de conocimientos les impedía expresarse con claridad y la interacción casi no se producía o resultaba totalmente artificial. En este sentido comprobamos que al permitir que los alumnos utilizasen la lengua materna mientras trabajaban en grupo, ganábamos en autenticidad y riqueza en el resultado de las tareas, sin menoscabo en la calidad de la comprensión y la producción en la lengua extranjera, ya que utlizaban constantemente dicha lengua a través de los documentos de apoyo. Además se exigió, en compensación, una mayor corrección lingüística en la realización de las tareas y en la presentación del trabajo individual.

Un factor que tuvimos que considerar, por experiencias previas en la aplicación de este enfoque, fue limitar la longitud y el alcance del proyecto para evitar rechazos de los alumnos al contenido de la tarea y al nivel lingüístico requerido para su realización. Para ello tuvimos que velar por la composición de los grupos, mezclando alumnos con diferentes habilidades: lingüísticas, artísticas o didácticas, de modo que, como ya hemos apuntado, todos se sintieran útiles.

Por último, quizá el punto más conflictivo de esta experiencia, para nosotras como profesoras, consistió en establecer unos criterios de evaluación, sobre todo porque al final el sistema educativo exige dar una calificación.

Por nuestra formación filológica tenemos tendencia a dar prioridad a la corrección lingüística e inconscientemente dejamos de lado otros criterios que pueden ser igualmente válidos. De este modo se plantea el dilema para el profesor: ¿Qué valor damos al trabajo realizado por un alumno con unas excelentes cualidades didácticas que le permiten «comunicar», pero que demuestra un mínimo nivel de conocimientos lingüísticos? ¿Cómo calificar a este alumno con respecto a otro que comunica sin problemas, por su excelente preparación lingüística, pero que no sabe implicar a los demás en una actividad por la falta de recursos didácticos?

Alcanzar ese difícil equilibrio que permite ponderar los diferentes factores de forma ecuánime e imparcial, constituye, a nuestro juicio, el punto culminante de la preparación y la responsabilidad que el Enfoque por Tareas exige al profesor.

\section{Referencias}

Breen, M. P. (1987). «Learner contributions to task design» en C. Candlin y D. Murphy (eds.). Language learning tasks. Englewood Cliffs. N J: Prentice Hall International.

BREEN, M. P. (1990). «Paradigmas actuales en el diseño de programas de lenguas» en Comunicación, lenguaje y educación. 7-8. pp. 7-32.

CaNale, M., y SWAIN, M. (1980). «Theoretical Basis of Communicative Approaches to Second Language Teaching and Testing». Applied Linguistics 1: pp. 1-47.

CANDIN, C. N. (1990). «Hacia la enseñanza de lenguas basada en tareas» en Comunicacion, Lenguaje y Educación. 7-8. pp. 33-53.

CARE, J. M., y DEBYSER, F. (1984). Simulations globales. Paris: BELC.

CEUlAR FuENTES, C. (1992). «El aprendizaje y la enseñanza de las lenguas modernas» en V. García Hoz (Dir.). Tratado de Educación Personalizada. Enseñanza y aprendizaje de las lenguas modernas. Madrid: Rialp. pp. 109-155. 
DeBYSER, F. (1986). L'Immeuble. París: Hachette-BELC.

ECHEvarRia, C. (1993). «Desarrollo del trabajo por tareas en el aprendizaje del inglés» en V. García Hoz (Dir.) Tratado de Educación Personalizada. Madrid: Rialp. pp. 189-206.

ESTAIRE, S., y ZANON, J. (1990). «El diseño de unidades didácticas mediante tareas: principios y desarrollo» en Comunicación, lenguaje y educación. 7-8, pp. 54-90.

FRIEDBOOTH, D. (1986). Projert Work. Oxford: Oxford University Press.

HAINES, S. (1989). Projects for the classroom. Nelson.

HOLEC, H. (1983). Autonomy and foreign language learning. Oxford: Pergamon.

Hymes, D. H. (1972). «On communicative competence» en J. B. Pride y J. Holmes (eds.) Sociolinguistics. Londres: Penguin Books.

Le Français dans le Monde n. ${ }^{\circ}$ 246, Janvier 1992. Spécial Jeux Olympiques: Les disciplines sportives. Ficha IV-VI. París: Hachette.

Ministerio de Educación y Ciencia (1992). Primaria. Area de Lenguas Extranjeras. Madrid: Ministerio de Educación y Ciencia.

NunaM, D. (1989). Designing Tasks for the Communicative Classroom. Cambridge: CUP.

PRABU, N. S. (1987). Secong language pedagogy: a perspective. Oxford: Oxford University Press.

REA, P. (1987). «Communicative curriculum validation: a task-based approach» en Candlin y Murphy (eds.) Language learning tasks. Lancaster Practical Papers in English Language Education, vol. 7. Londres: Prentice Hall.

SEFARINI, D. (1989). «L'Hôtel: une simulation fonctionnelle» en Le Français dans le Monde $n{ }^{\circ} 228$. PP. 57-91.

VAN EK, J. A. (1988). Objectifs de l'apprentissage des langues vivantes: v.I. Contenus et portée. Strasbourg: Conseil de l'Europe.

\section{El enfoque por tareas en la enseñanza/aprendizaje} del francés lengua extranjera: una experiencia para la reflexión

$$
\begin{aligned}
& \text { Marienma García, Concha Prieto y } M^{a} .^{a} \text { Jesús Santos } \\
& \text { CL\&E, 1994, 24, pp. 71-78 }
\end{aligned}
$$

Resumen: En este artículo presentamos una experiencia de enseñanza/aprendizaje del francés, lengua extranjera, siguiendo una metodología basada en el enfoque por tareas.

En primer lugar, abordamos el marco teórico en el que se incluye dicha experiencia, estableciendo los principios y componentes del enfoque. Seguidamente desarrollamos la unidad temática tal y como se llevó a la práctica, con un análisis previo de las necesidades e intereses del público al que se dirigió. Concluimos con una reflexión sobre el trabajo realizado, señalando los aspectos positivos y las limitaciones con las que nos encontramos.

Datos sobre el autor: Las autoras de este artículo son profesoras en la Escuela Universitaria de Educación de Palencia y pertenecen al Departamento de Didáctica de la Lengua y la Literatura (Francés) de la Universidad de Valladolid. Mariemma García Alonso trabaja sobre terminología didáctica y técnicas de animación a la lectura. Concepción Prieto Rodriguez está investigando en el análisis del discurso en los libros de texto y Maria Jesús Santos Maldonado investiga sobre el condicionamiento de la lengua materna en la enseñanza/aprendizaje de una lengua extranjera.

Dirección: Universidad de Valladolid. Escuela Universitaria de Formación del Profesorado. C/ Prolongación de la Ojeda, s/n, 34006 Palencia

(C) PERMISOS PARA CITAR O REPRODUCIR EN OTRAS FUENTES: Se pueden citar libremente hasta 500 palabras. Para reproducir una porción de texto mayor, figuras o ilustraciones, se deberá pedir permiso por escrito a la revista, especificando el uso al que se destina el texto. En todos los casos, se deberá citar el copyright de $C L \& E$. En el caso de artículos o textos que hayan sido a su vez reproducidos en $C L \& E$ los interesados deberán dirigirse tanto a los detentadores del copyright original como a $C L \& E$, en el caso de que se quiera hacer uso de la traducción. FOTOCOPIAS: Para todo lo relacionado con el uso mediante fotocopia del material de esta revista, deberán dirigirse a: CEDRO, C/ José Marañón, 10, 3..$^{\circ}$ Izda. Tel. 594 15 75. Fax 4453567 